\title{
XXVI. International Conference on Coordination Chemistry (26th ICCBiC), June 2017, Slovakia
}

\author{
Peter Segl'a ${ }^{1} \cdot$ Miroslav Tatarko ${ }^{1} \cdot$ Marián Valko $^{2}$
}

Published online: 2 February 2018

(c) Institute of Chemistry, Slovak Academy of Sciences 2018

The XXVI. International Conference on Coordination and Bioinorganic Chemistry (ICCBiC) was held from June 4 to June 9, 2017, in the Smolenice Castle, Slovakia. The conference was organized by the Slovak Chemical Society, the Slovak Academy of Sciences, and Slovak University of Technology in Bratislava. The founder of the traditional ICCBIC conferences in 1964 was Prof. Ing. Ján Gažo, DrSc. (1929-1983) and in his honour the organizing committee of the ICCBIC suggested and the Slovak Chemical Society established the "Ján Gažo Award", consisting of the "Ján Gažo Medal" and the certificate of the Slovak Chemical Society in the form of a diploma.

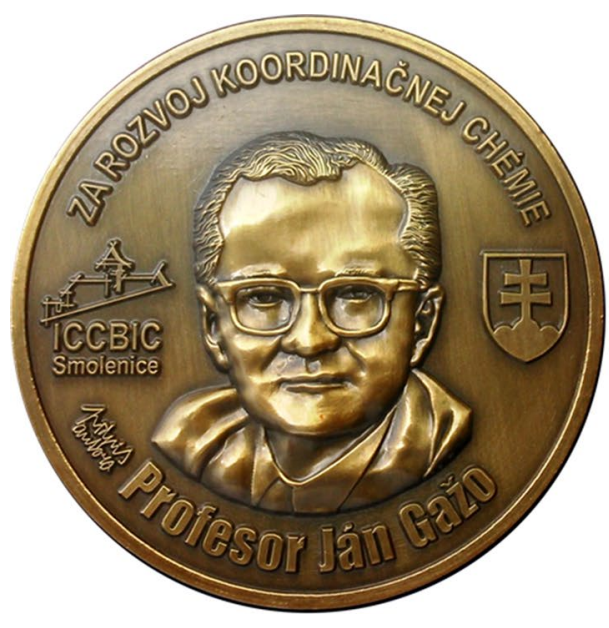

Marián Valko

marian.valko@stuba.sk

Peter Segla

peter.segla@stuba.sk

Miroslav Tatarko

miroslav.tatarko@stuba.sk

1 Department of Inorganic Chemistry, Slovak University of Technology in Bratislava, Radlinského 9, 81237 Bratislava, Slovakia

2 Department of Physical Chemistry, Slovak University of Technology in Bratislava, Radlinského 9, 81237 Bratislava, Slovakia
The first medal was awarded to Prof. Ing. Gregor Ondrejovič, DrSc. at the official opening ceremony of the conference, on June 4, 2017. After the sudden death of Prof. Gažo in 1983, Prof. Ondrejovič continued to organize regular conferences with his colleagues from the Department of Inorganic Chemistry, STU, in 1983-2000.

This year, 72 scientists from abroad and 38 scientists from Slovakia participated in the conference; geographically, 22 countries from four continents were represented. Active participation of 43 colleagues younger than 35 years is a clear sign of the challenging future of both coordination chemistry and the series of ICCBiC. The participants have

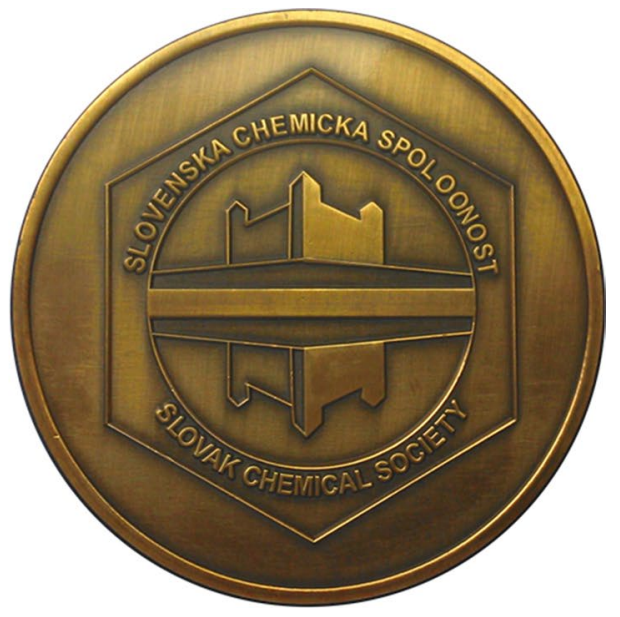

reported their scientific results in 90 lectures (of them 27 lectures were given in the "Young Scientists Session") and in 16 posters.

The topic of plenary lecture in section "Electronic molecular and crystal structures" was: "Coordination self-assembly: from the origins to the latest advances", presented by Prof. Makoto Fujita, University of Tokyo, Japan. Molecular self-assembly based on coordination chemistry has made an explosive development in recent years. Over the last more than 25 years, have been showing that the simple combination of transition-metal's square planar geometry with 
pyridine-based bridging ligands gives rise to the quantitative self-assembly of nano-sized, discrete organic frameworks.

In section "Solution and solid state reactivity" invited plenary lecture was: "Quantum information with molecular nanomagnets" presented by Dr. Floriana Tuna, University of Manchester, United Kingdom. Quantum information processing (QIP) offers one of the most exciting challenges in the study and development of molecular materials. Instead of operating with classical bits of information, a quantum computer will work with quantum bits (known as qubits). The implementation of such an advanced technology demands chemical systems with robust quantum states that can be individually controlled and measured, and are able to sustain coherent quantum manipulations.

Finally, in section "Applied inorganic and coordination chemistry" invited plenary lecture was: "If coordination chemistry meets quantum physics: metal complexes as nuclear spin qubits" presented by Prof. Mario Ruben, Institute of Nanotechnology and Institute of Inorganic Chemistry, KIT, Karlsruhe, Germany. Magnetic metal complexes have been proposed as Qubits candidate units for Quantum Computing (QC) and Quantum Information Processing.

All contributions on ICCBiC have been published in the Conference book of abstracts. Also a monograph (13th in the series)_- "Modern Trends in Coordination, Bioinorganic, and Applied Inorganic Chemistry", comprising 15 original contributions based on conference lectures has been published in the form of storage medium (USB key). Moreover, another 11 full papers will appear in a second special issue of Chemical Papers (CC journal, Springer).

The organizing committee very much appreciates the contribution of all participants to the smooth running, success and results of the conference. It is necessary to thank the participating prominent coordination chemists who keep the high scientific level of the conference.

The next conference in this series, XXVII. ICCBiC will be held on July 2-7, 2019 again in Smolenice Castle, Slovakia. We look forward to meeting you there.

\section{Special issue of chemical papers}

The special issue of Chemical Papers from the XXVI International Conference on Coordination and Bioinorganic Chemistry held in June 2017 in Smolenice, Slovakia, presents papers covering a wide range of studies dealing with the most recent developments in coordination and bioinorganic chemistry. In total, one review paper and ten original contributions have been submitted.

Brachnakova and Salitros present a review paper on ligand-driven light-induced spin transitions in compounds possessing spin-crossover phenomenon. The Review covers the most interesting iron and cobalt spin-crossover compounds with photoisomerizable ligands and provides the overview of the most important findings based on the ligand-driven light-induced spin change effect.

Original paper by Bortoluzzi and collaborators reports on photoluminescence features of europium and terbium derivatives with conjugate base of malondialdehyde. The results have shown that conjugate base of malondialdehyde is an efficient antenna-ligand for the sensitization of $\mathrm{Tb}(\mathrm{III})$ luminescence.

Scrivanti and coworkers reported the synthesis of a novel type of bidentate triazolyl-oxazoline ligand and its yttrium and lanthanide complexes. The coordination mode of the ligand was described by means of DFT calculations. In addition, photoluminiscent properties of prepared complexes were studied.

Starikova reported computational studies of trinuclear heterometallic complexes of iron(II) 2,6-di(pyrazol-1-yl) pyridine chelates with 1,3,5-triazapentadiene metal $(\mathrm{M}=\mathrm{Co}$, $\mathrm{Ni}, \mathrm{Cu}, \mathrm{Zn}$ ) containing linkers. This study explored that the variation of metal atoms of bischelate linker underwent complete $(\mathrm{M}=\mathrm{Co}, \mathrm{Ni})$ and partial $(\mathrm{M}=\mathrm{Cu}, \mathrm{Zn})$ two-step spincrossover. In addition,.it has been concluded that the weak exchange interactions between paramagnetic centers make trinuclear complexes with nickel- and copper-containing linkers III ( $\mathrm{M}=\mathrm{Ni}, \mathrm{Co})$ promising precursors for the design of molecular electronics devices.

Starikov and coworkers reported DFT study of the oligomers of $\mathrm{Co}(\mathrm{II}), \mathrm{Ni}(\mathrm{II}), \mathrm{Cu}(\mathrm{II})$ and $\mathrm{Zn}$ (II) $\beta$-diketonates and their stability towards dissociation into monomeric units. The study has shown that the DFT method complemented with CAM and/or D3BJ corrections is suitable for accounting dispersion interactions and provides insight into the structural characteristics of studied oligomers.

Hand and his group reported on the synthesis, structure and properties of dinuclear Rh(II) complex. In this study, cis- $\left[\mathrm{Rh}_{2}(4-\mathrm{Me}-\mathrm{pf})_{2}\left(\mathrm{O}_{2} \mathrm{CCMe}_{3}\right)_{2}\right]$ was newly synthesized from cis- $\left.\left[\mathrm{Rh}_{2} \text { (4-Me-pf }\right)_{2}\left(\mathrm{O}_{2} \mathrm{CCF}_{3}\right)_{2}\right]$ (4-Me-pf- = N,N'-dip-tolylformamidinate anion) by a trifluoroacetato $\rightarrow$ pivalato ligand substitution reaction. The structure of the complex was obtained by X-ray crystal structure analysis. Absorption bands for both complexes were reasonably assigned using TD-DFT calculations. In addition, it was confirmed that the electron-donating t-butyl groups on the carboxylato bridges give rise to negative shifts in the redox potentials.

Mikurya and coworkers prepared tetranuclear mixedvalent manganese complexes with pentadentate Schiff-base ligands having a Y-shaped core. Complexes were characterized by X-ray crystallography, electronic spectroscopy and 
magnetic measurements which revealed an antiferromagnetic interaction of these complexes.

Segla and coworkers prepared a series of monomeric and dimeric carboxylato copper(II) complexes with 4-pyridinemethanol and isonicotinamide. The complexes were characterized by electronic, IR, EPR spectroscopy, magnetic susceptibility and X-ray crystallography. Structural studies explored a distorted tetragonal-bipyramidal environment around the copper ion for monomeric complexes. Two dimeric copper(II) complexes consist of units of the known paddle-wheel dicopper(II) tetracarboxylates. The complex molecules of all compounds are connected through hydrogen bonds into supramolecular chains.

Smolko and coworkers reported on the tetracoordinate cobalt(II) complexes with neocuproine. The results have shown that the complexes behave as single-molecule magnets in an applied external field. Obtained results indicate that all cobalt(II) complexes are able to bind to the CT DNA via intercalation. In addition, the Topo I inhibition studies revealed that all three $\mathrm{Co}$ (II) complexes are able to completely inhibit this enzyme at concentration of $45 \mu \mathrm{M}$.

Khattak and coworkers studied kinetics of the reduction of dicyanobis(phen)iron(III) by acetylferrocene and methylferrocenemethanol. The results obtained from the kinetic data and its thermodynamic aspect refer to the existence of equilibrium between protonated and unprotonated acetylferrocene, and also $\alpha$-methylferrocenemethanol. The unprotonated and protonated species of these compounds take part in the electron transfer process, and donate electron to dicyanobis(phen)iron(III). This study confirmed that the rate of reaction of the subject compounds is controlled by hydroxyl-containing compounds such as saturated $\alpha$-methylferrocenemethanol or unsaturated conjugate acid of acetylferrocene. This led to the conclusion that the protonation of these compounds changes their reactivity in chemical and biochemical processes either partially or completely, leading to significant changes in the mechanism.

Ziegenheim and coworkers synthesized $\mathrm{Cu}(\mathrm{II}) \mathrm{Cr}(\mathrm{III})-$ $\mathrm{L}$ (ayered) $\mathrm{D}$ (ouble) $\mathrm{H}$ (ydroxide) and studied its catalytic and intercalating properties. The obtained $\mathrm{CuCr}-\mathrm{LDH}$ proved to be a good host for the intercalation of 1-prolinate with the direct anion-exchange method applying aqueous acetone as the reaction medium. The pristine $\mathrm{CuCr}-\mathrm{LDH}$ was unsuitable for the catalysis of allylic alcohol oxidation; however, the heat-treated derivative, when the layered structure was destroyed, performed rather well in the (exclusive) epoxidation of this molecule. 